\title{
Transverse emittance preservation during bunch compression in the Fermi free electron laser
}

\author{
S. Di Mitri, ${ }^{*}$ E. M. Allaria, P. Craievich, W. Fawley, L. Giannessi, A. Lutman, G. Penco, S. Spampinati, and M. Trovo \\ Sincrotrone Trieste, Trieste, Italy
}

(Received 28 July 2011; published 13 February 2012; publisher error corrected 16 February 2012)

\begin{abstract}
A characterization of the transverse emittance of a $200 \mathrm{pC}, 6$ ps long electron bunch has been performed in the Fermi@Elettra Free Electron Laser (FEL) first bunch compressor area. This region includes a magnetic bunch length compressor, diagnostics, and quadrupole magnets. The beam is time compressed in one stage, without linearization of the longitudinal phase space. Some growth of the normalized emittance has been measured in the compressor area. To understand this effect, we have investigated mechanisms of emittance growth such as coherent synchrotron radiation emission, chromatic aberration, and spurious dispersion. We show that careful optics control inside the compressor is essential for emittance growth reduction. The final configuration of one-stage magnetic compression limits the emittance to values below the design goal of $2 \mathrm{~mm} \mathrm{mrad}$, up to a compression factor of about 5 . This machine configuration has been adopted to optimize FEL radiation output at wavelengths in the range 30-60 nm.
\end{abstract}

DOI: 10.1103/PhysRevSTAB.15.020701

PACS numbers: 29.27.Bd, 41.85.-p, 29.20.Ej

\section{INTRODUCTION}

The Fermi@Elettra free electron laser (FEL) at the Elettra Laboratory of Sincrotrone Trieste [1] is a major European FEL project. With its high peak brilliance, extreme ultraviolet to soft $\mathrm{x}$-ray pulses, Fermi will become the international user facility in Italy for scientific investigations of fast and high resolution processes in material science and physical biosciences. Fermi is a single-pass, S-band linac-based externally seeded FEL implementing high gain harmonic generation [2-5] in the 100-4 nm fundamental output wavelength range. The layout is shown in Fig. 1. Table I lists the main machine and electron beam parameters adopted during commissioning.

Commissioning of Fermi started in September 2009, the first FEL output with seeded operation was produced in December 2010, and first light was provided to users in April 2011 [6]. Although the nominal configuration includes two stages of magnetic bunch length compression, only the first one, called $\mathrm{BC} 1$, was installed at the time of the measurements reported in this paper. $\mathrm{BC} 1$ is a movable chicane that allows a continuously tunable bending angle in the range $0-122 \mathrm{mrad}$. The maximum $\left|R_{56}\right|$ term provided by the system is $96 \mathrm{~mm}$. The linear theoretical bunch length compression factor is defined as $C=1 /\left(1+h R_{56}\right)$, where $h=d E /(E d z)$ is the linear energy chirp relative to the reference energy. A short rf structure with the accelerating field at the fourth harmonic of the $3 \mathrm{GHz}$ linac shall be used to linearize the particle longitudinal phase space during compression [7]. In the present operation, this

\footnotetext{
*simone.dimitri@elettra.trieste.it

Published by the American Physical Society under the terms of the Creative Commons Attribution 3.0 License. Further distribution of this work must maintain attribution to the author(s) and the published article's title, journal citation, and DOI.
}

structure is not yet installed and the compression linearity is limited by the sinusoidal rf time curvature and the chicane second order path-length dependence on the particle energy. For these reasons, the compressed electron bunch current profile is characterized by a region at the bunch head with several hundreds of amperes, followed by a long tail with $\sim 100 \mathrm{~A}$ at its end.

Although particle tracking simulations do not reveal any critical emittance blowup in $\mathrm{BC} 1$ for compression factors equal to or smaller than 10 [1], some growth of the horizontal normalized emittance, $\varepsilon_{N, x}$, has been observed during commissioning. In the following, we will always refer to the normalized emittance, unless specified otherwise. Because of the importance of preserving the transverse emittance along the entire machine, a campaign of projected emittance measurements has been carried out to understand and recover the discrepancy between the model predictions and the experimental results. In particular, the impact of spurious dispersion, chromatic aberration, and coherent synchrotron radiation (CSR) emission [8-12] has been studied. We anticipate that a comparison of the experimental results with theoretical and simulation predictions identifies two main sources of horizontal emittance dilution: spurious dispersion at compression factors smaller than $\sim 5$ (L1 S-band rf phase $\Delta \phi<20^{\circ}$ far from crest) and CSR emission at larger compression factors $\left(\Delta \phi \geq 20^{\circ}\right)$. So far, we have been ignoring the effect of geometric transverse wakefield on the projected emittance. Detailed studies [13] show that the single bunch, head-tail lateral displacement can be neglected in the early part of the linac, under the assumption of a machine alignment within the specifications $[1,13]$.

Emittance studies during magnetic compression in the presence of nonlinear longitudinal dynamics have already been published [14-17], but only for lower energy $(<60 \mathrm{MeV})$, lower brightness $\left(\varepsilon_{N, x}>6 \mathrm{~mm} \mathrm{mrad}\right)$, and 


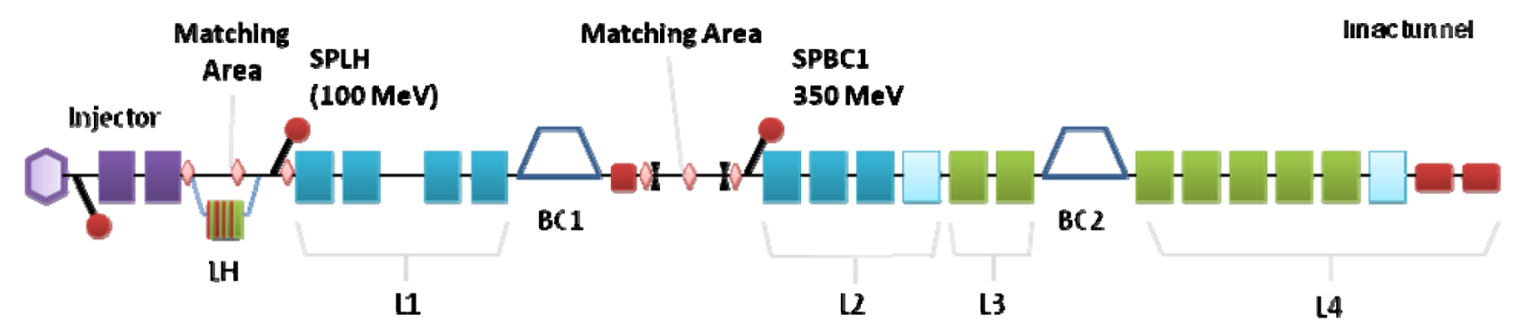

FEL1:1 modulator +1 dispersive section +6 radiators

undukatorhal

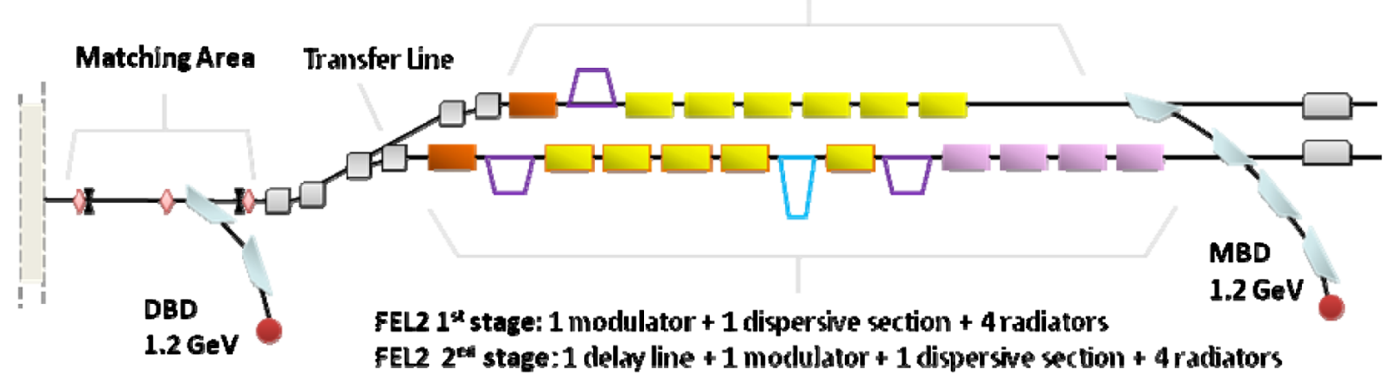

FIG. 1. Split layout of the Fermi FEL (conceptual, not to scale). The linac includes the injector, the laser heater (LH) area, different types of accelerating structures—represented with different colors—accommodated in linac 1 (L1), linac 2 (L2), linac 3 (L3), and linac 4 (L4), two magnetic chicanes for bunch length compression (BC1 and BC2), three optics matching area, the laser heater spectrometer line (SPLH), the first compressor spectrometer line (SPBC1), and the diagnostic beam dump (DBD). A transfer line brings the electron beam to the FEL1 or FEL2 undulator line (different undulator segments are represented by different colors), followed by the main beam dump (MBD) line.

even higher charge beams ( $\geq 5 \mathrm{nC}$ ). Exhaustive studies as in $[7,8]$ deal with beam parameters close to those discussed in this article, but they are focused on the CSR-induced emittance growth in presence of a linearized longitudinal phase space. An analysis of slice emittance dynamics in the presence of a two-stage, nonlinear magnetic compression can be found in [18], where a projected emittance of approximately $8 \mathrm{~mm}$ mrad has been achieved. In this paper, we addressed the problem of preserving the normalized emittance during one-stage, nonlinear magnetic compression in a high brightness, linac-driven FEL facility. As a result of our study, an alternative configuration of the onestage magnetic compression has been found that minimizes

TABLE I. Fermi electron beam and machine parameters for one-stage compression in BC1.

\begin{tabular}{lcc}
\hline \hline Parameter & Value & Units \\
\hline Charge & 200 & $\mathrm{pC}$ \\
Initial bunch length, FWHM & 6 & $\mathrm{ps}$ \\
Pulse shaping & flat & \\
Initial normalized emittance, rms & $1.5-2.0$ & $\mathrm{~mm} \mathrm{mrad}$ \\
BC1 bending angle & 85 & $\mathrm{mrad}$ \\
BC1 $R_{56}$ & -40.9 & $\mathrm{~mm}$ \\
L1 rf voltage, peak & 250 & $\mathrm{MV}$ \\
Mean energy at BC1, on-crest & 350 & $\mathrm{MeV}$ \\
Linear compression factor for $\Delta \phi=25^{\circ}$ & 6.5 & \\
\hline \hline
\end{tabular}

the projected emittance growth of a $200 \mathrm{pC}$ beam to below the design goal of $2 \mathrm{~mm}$ mrad, up to a compression factor of about 5. This basic compression scheme has been adopted for the seeded Fermi FEL lasing at output wavelengths in the range 30-60 $\mathrm{nm}$ [6].

The article is organized as follows. Section II describes the $\mathrm{BC} 1$ area layout and the method for projected emittance measurement. Section III depicts the analytical and simulation tools that have been used to predict emittance growth in the presence of spurious dispersion, chromatic aberration, and CSR emission. Section IV shows the measurements of emittance as a function of the linac rf and magnetic lattice setting. Section V summarizes our conclusions, followed by an Appendix about the reconstruction of the particle energy distribution during compression.

\section{TOOLS FOR EMITTANCE MEASUREMENT AND OPTICS CONTROL}

Emittance measurements are routinely performed in the $\mathrm{BC} 1$ area via the quadrupole scan technique [18]. The layout is sketched in Fig. 2.

The emittance measurement involves a scan over at least 9 different values of the quadrupole strength and 5 images of the beam spot in the transverse plane per quadrupole setting. The postprocessing of the images to determine the beam size standard deviation is a critical step of the measurement process [19]. A background signal, acquired with 


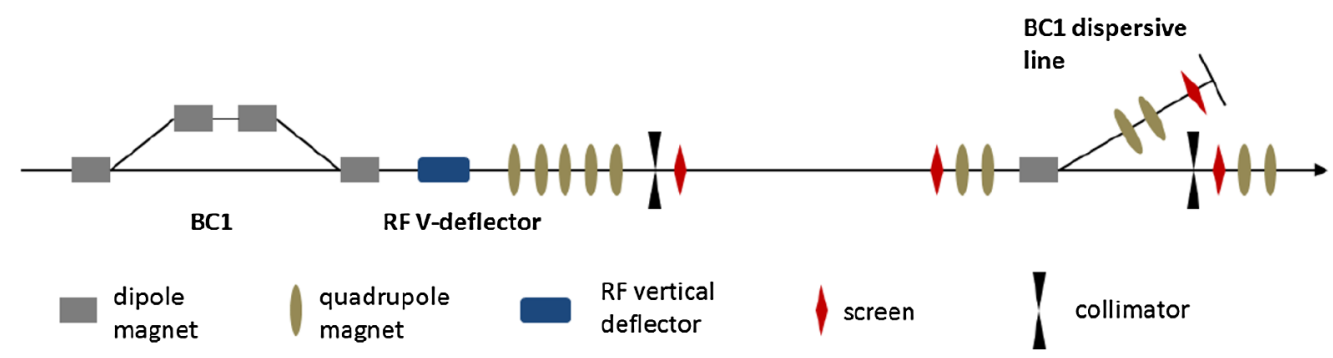

FIG. 2. Layout of the Fermi@Elettra BC1 area (conceptual, not to scale). The last quadrupole magnet of the group of 5 downstream of $\mathrm{BC} 1$ is used for the beam size scan and emittance measurement.

no electron beam at the screen (due to a closed photoinjector laser shutter), is subtracted from each recorded beam spot image. Different approaches to compute the beam size standard deviation are available; i.e., we can (i) compute the raw rms over the entire profile, (ii) compute the raw rms of a 5\% area-integrated projection cut [20], (iii) fit the image projection with a Gaussian, (iv) with an asymmetric Gaussian, or (v) with an ad hoc function described here. This function is built on the basis of the "Super Gaussian" function described in [21] with the addition of a fitting parameter to evaluate the asymmetry of the profile:

$$
Y=K \cdot \exp \left(\frac{|x-\mu|^{a+b \cdot \operatorname{sign}(x-\mu)}}{g+d \cdot \operatorname{sign}(x-\mu)}\right) \quad \text { with } K=\frac{1}{(g+d)^{1 /(a+b)} \Gamma(1+1 / a+b)-(g-d)^{1 /(a-b)} \Gamma(1+1 / a-b)},
$$

where $\mu, a, b, g$, and $d$ are fitting parameters. When $a=2$ and $b=0$, the function is a Gaussian with asymmetric standard deviation for the left and right tail. The implementation of such a function allows us to improve the fitting of the projected beam size during the quadrupole scan in case of strongly non-Gaussian profiles, particularly with regards to drawn out tails. Usually, this results in a computed beam size and emittance value higher than that of a simple Gaussian fit. However, since the FEL performance relies on the optimization of the electron bunch core, we use the Gaussian fit more often in the optics matching procedure, while the ad hoc function is applied to measure the emittance due to the entire particle ensemble.

The quadrupole scan diagnostic system uses either an optical transition radiation (OTR) or a yttrium aluminum garnet (YAG) target screen, on which the beam transverse profile is projected. The resolution of the spot size measurement is $20 \mu \mathrm{m}$ with the OTR, $30 \mu \mathrm{m}$ with the YAG. Unfortunately, the OTR cannot be used for compression factors larger than $\sim 3$ because contamination signals of coherent OTR (most likely due to longitudinal microbunching) start appearing, saturating the image and seriously affecting the beam size measurement [22].

In the absence of such contamination, a beam size jitter much smaller $(\leq 10 \%)$ than the diagnostic resolution is typically measured. Error propagation as described in [19] leads to an error of few percent on the central value of the Twiss parameters and projected emittance. During some measurements, we have observed even larger beam size shot-to-shot jitter, leading to larger error bars. Although we suspect some rf instabilities play a role, in the case of measurements affected by large jitter we have decided to adopt the largest beam size error for all points, in order to present a conservative scenario of the measurement accuracy.

The beam optics matching and transport is performed in Fermi with the ELEGANT code [23], which obtains experimental data via an interface to the Tango server [24] through MATLAB [25] scripts. The procedure begins by measuring the beam Twiss parameters at the entrance of the quadrupole magnet that is used for the emittance scan. Then, ELEGANT computes the quadrupole strengths required for matching the beam to the design optics. Via a MATLAB/Tango script, these strengths are finally applied to the machine. The betatron mismatch parameter [26] is

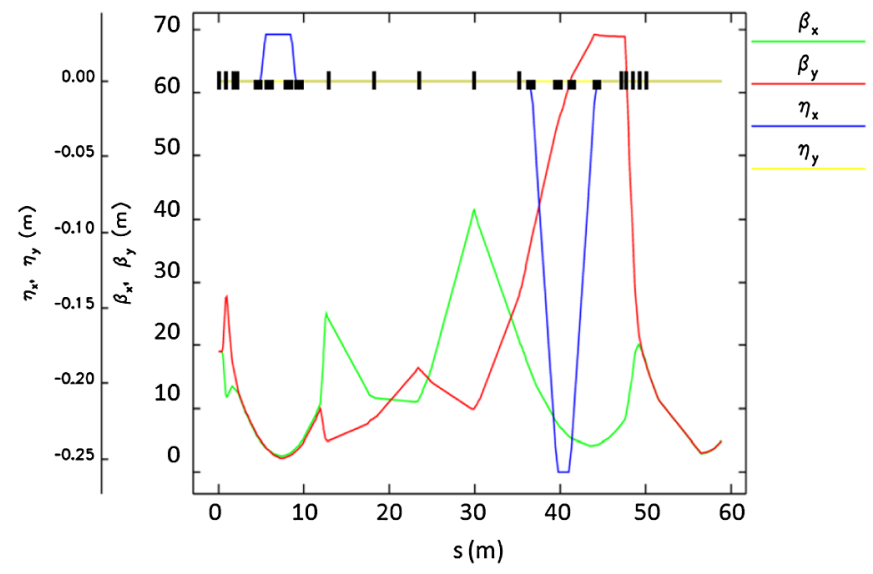

FIG. 3. Design optics from the $\mathrm{LH}$ to the $\mathrm{BC} 1$ area (the negative horizontal dispersion is inside $\mathrm{BC} 1$ ). The small upright rectangles represent quadrupole magnets while squares are dipole magnets. 
usually measured after the matching procedure and confirms that the beam optics is normally well matched after 2 or 3 iterations. Initially, we match the transport optics from the exit of the photoinjector through the first accelerating section L0 to the so-called laser heater (LH) area corresponding to the beam energy of $100 \mathrm{MeV}$. Then, the beam is transported to the $\mathrm{BC} 1$ area with a mean energy of $350 \mathrm{MeV}$ when the next linac section L1 is run on-crest. The design optics from the $\mathrm{LH}$ to the $\mathrm{BC} 1$ area is shown in Fig. 3.

\section{MODELING}

In this section we consider the possible impact of a spurious dispersion upon the projected emittance. We presume that the geometric and the chromatic contributions add quadratically to obtain the effective beam size standard deviation. Consequently, we compute the effective growth of the emittance in the presence of particle chromatic motion relative to the purely geometric case:

$\sigma=\sqrt{\varepsilon \beta+\left(\eta \sigma_{\delta}\right)^{2}} \equiv \sqrt{(\varepsilon+\Delta \varepsilon) \beta} \Rightarrow \frac{\Delta \varepsilon}{\varepsilon}=\frac{\left(\eta \sigma_{\delta}\right)^{2}}{\varepsilon \beta}$.

We have adopted the following notation: $\sigma$ for the beam size; $\sigma \delta$ for the energy spread; $\beta$ and $\eta$ for the betatron and dispersion functions, respectively, in the same transverse plane in which the geometric emittance, $\varepsilon$, is defined. For a situation with an unperturbed normalized emittance equal to $1.5 \mathrm{~mm}$ mrad, relative energy spread of $1 \% \mathrm{rms}$ (a measurement of the energy spread during compression and its comparison with the model is given in the Appendix) at the mean energy of $330 \mathrm{MeV}$, horizontal betatron function of $3 \mathrm{~m}$, and spurious dispersion of $4 \mathrm{~mm}$ (this particular choice is discussed in the next section), we expect a relative emittance growth of $24 \%$.

The effect of chromatic aberration induced by magnetic focusing may be analytically computed as the determinant of the beam matrix [27] affected by a quadrupole kick error $\Delta x^{\prime}=k l x \delta$, where $k l$ is the normalized quadrupole integrated strength, $x$ is the particle distance from the quadrupole center, and $\delta$ is the relative energy deviation. We consider a maximum normalized integrated strength $k l=$ $0.5 \mathrm{~m}^{-1}$ over the entire $\mathrm{LH}$ and the $\mathrm{BC} 1$ area. Assuming a Gaussian particle distribution in the transverse phase space, we have

$$
\frac{\Delta \varepsilon}{\varepsilon} \cong \frac{1}{2}\left(\beta k l \sigma_{\delta}\right)^{2} \leq 1 \%
$$

for $\sigma_{\delta}=1 \%$ and $\beta \leq 30 \mathrm{~m}$ in the horizontal plane.

The emittance growth due to CSR in the $\mathrm{BC} 1$ bending magnets can be estimated in a similar way as in Eq. (2). The kick $\Delta x^{\prime}$ is now proportional to the horizontal dispersion in $\mathrm{BC} 1$ and the CSR-induced energy deviation. According to the Fermi commissioning parameters (see Table I), we see that for compression factors $C \leq 6.5$ the bunch length, $l_{b}$, is longer than the CSR slippage length
[11], $s_{L} \cong \frac{R \theta^{3}}{24}$, where $R$ and $\theta$ are the $\mathrm{BC} 1$ bending radius and angle, respectively. At the same time, the retarded bending angle is $\gamma \theta \gg 1$. Thus, according to [11], the electron beam is in the so-called long bunch, long magnet CSR regime for which the total CSR energy loss for a longitudinally uniform charge distribution is

$$
\Delta E_{\mathrm{CSR}} \cong-\frac{1}{4 \pi \varepsilon_{0}} \frac{Q^{2}}{l_{b}}[4 \ln (\gamma \theta)-2],
$$

where $Q$ is the bunch charge. This formula neglects any transverse variations in CSR loss (the "1D" limit). The relative emittance growth predicted by the beam matrix formalism for the beam parameters of Table I is then

$$
\frac{\Delta \varepsilon}{\varepsilon} \cong \frac{1}{2} \frac{\beta}{\varepsilon}\left(\theta \sigma_{\delta, \mathrm{CSR}}\right)^{2} \cong 4 \% .
$$

The energy spread standard deviation, $\sigma_{\delta \text {, CSR }}$, is a fraction of the total energy loss in Eq. (4) and is estimated as follows. According to [11], the average particle energy loss for the aforementioned CSR regime is proportional to the logarithmic term in Eq. (4) and to the local current density; in our case the latter can be described by a ramp (see later in this section). Accordingly, the CSR-induced energy loss along the bunch can be described by a linear energy chirp. We make the approximation that the particle energy distribution associated to such a chirp is a uniform distribution, whose standard deviation is $\sigma_{\delta, \mathrm{CSR}}=$ $\sqrt{12} \Delta E_{\mathrm{CSR}}$. Our approach should be sufficient for a rough estimate of the CSR effect on the emittance. However, several other approximations are implicit in Eq. (5). It assumes a CSR-induced perturbation kick $\Delta x^{\prime}=$ $\eta^{\prime} \sigma_{\delta \text {,CSR }}$ given at the end of the last dipole magnet of $\mathrm{BC} 1$, where for a small bending angle $(\theta \ll 1)$ we have $\eta^{\prime} \approx \theta$. The design betatron function at this point is $6 \mathrm{~m}$. It neglects CSR effects along the first three dipole magnets of the chicane as well as any local correlation between the energy spread induced by CSR with the longitudinal bunch coordinate and, moreover, how such a correlation might

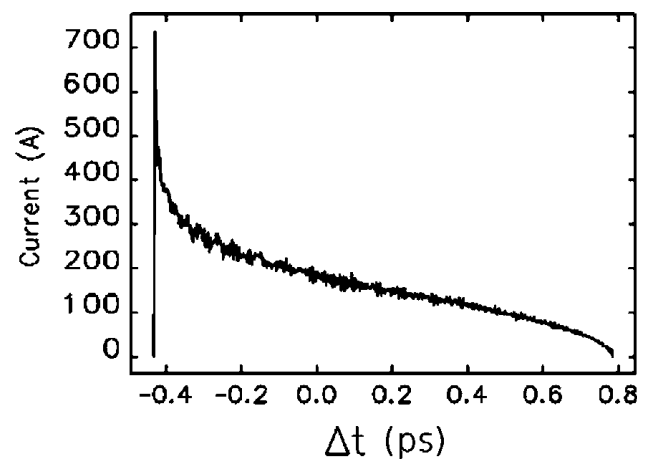

FIG. 4. Current profile after compression in BC1. $C=6.5$ is achieved with $\mathrm{BC} 1$ at $85 \mathrm{mrad}$ bending angle, $\mathrm{L} 1 \mathrm{rf}$ phase at $25^{\circ}$ far from crest. ELEGANT simulation result including CSR and LSC. 
TABLE II. Mechanisms of emittance growth and their theoretical strength.

\begin{tabular}{lrc}
\hline \hline $\begin{array}{l}\text { Mechanism of } \\
\text { emittance growth }\end{array}$ & $\Delta \varepsilon / \varepsilon$ & Notes \\
\hline Spurious dispersion & $24 \%$ & $\sigma_{\delta}=1 \%, \eta_{x}=4 \mathrm{~mm}, \beta_{x}=3 \mathrm{~m}$ \\
Chromatic aberration & $1 \%$ & $\sigma_{\delta}=1 \%, \beta_{x}=30 \mathrm{~m}$ \\
CSR & $4 \%$ & $\sigma_{\delta}=1 \%, C=6.5$ \\
\hline \hline
\end{tabular}

translate into projected emittance. To check the accuracy of these assumptions, we benchmarked the prediction of Eq. (5) with a $5 \times 10^{6}$ macroparticle tracking simulation performed with ELEGANT. We will show in the next section that ELEGANT predicts almost the same amount of emittance growth. Figure 4 shows the ELEGANT-predicted current profile after compression in $\mathrm{BC} 1$ by a factor 6.5. High order transport matrices are used to take into account optical aberrations, but neither geometric coupling nor spurious dispersion is included. CSR and longitudinal space charge (LSC) [28-30] are implemented as 1D impedances [31]. Previous studies [32,33] indicate that the 1D CSR and LSC models are accurate enough to describe the real particle dynamics in the Fermi linac. We summarize in Table II the aforementioned sources of emittance growth and their theoretical strengths.

\section{EXPERIMENTS}

\section{A. Emittance as a function of rf phase}

When $\mathrm{BC} 1$ is activated at the bending angle of $85 \mathrm{mrad}$, the electron bunch length is compressed by moving the L1 rf phase away from the nominal crest phase of $90^{\circ}$. The crest phase is determined empirically by the condition of maximum beam energy, as measured in the spectrometer line downstream of $\mathrm{BC} 1$ (see Fig. 2). The compression factor reported in the following is computed analytically in the linear approximation, but is based upon the real machine setup.

At first, in order to separate CSR effects from any other chromatic mechanism of emittance degradation, we measured $\varepsilon_{N, x}$ as a function of the energy spread in the $\mathrm{BC} 1$ area, with $\mathrm{BC} 1$ at zero bending angle. Figure 5 shows that $\varepsilon_{N, x}$ is approximately preserved for a large excursion of the rf phase $\left(|\Delta \phi|<20^{\circ}\right.$ from the crest) and, within this range, in good agreement with the simulation. In another set of measurements, we moved $\mathrm{BC} 1$ to $85 \mathrm{mrad}$ bending angle and determined $\varepsilon_{N, x}$ over a large range of L1 $\mathrm{rf}$ phase. As shown in Fig. 6, the maximum emittance value, expected at the point of full compression, is obtained at approximately the same rf phase in the experimental measurement $\left(\phi=119^{\circ}\right)$ and in the numerical simulation $\left(\phi=120^{\circ}\right)$. During the measurement we found that the initial emittance value is approximately recovered in overcompression mode $\left(\phi>120^{\circ}\right)$. We finally note that for $C=6.5\left(\phi=115^{\circ}\right)$, ELEGANT predicts a relative

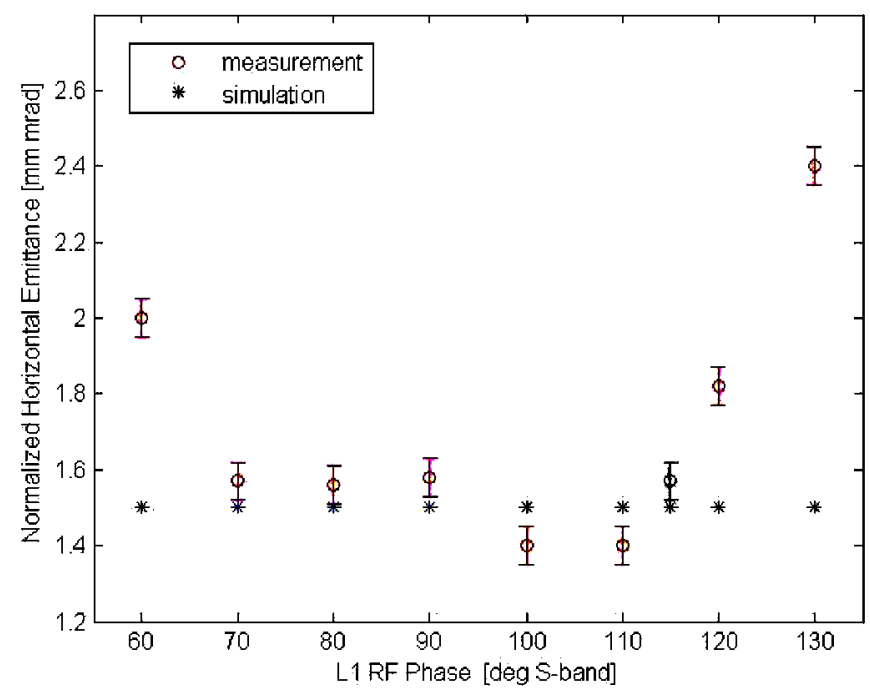

FIG. 5. Comparison of the normalized horizontal emittance measured and simulated as a function of the L1 rf phase. BC1 is set straight. The maximum beam energy is for $90^{\circ}$. A relative emittance growth smaller than $1 \%$ is predicted by particle tracking over the range of $\mathrm{rf}$ phases considered here.

emittance growth of $3 \%$ that is in good agreement with the analytical estimation in Eq. (5).

However, the experimental and simulation data show a discrepancy in Fig. 5 at large off-crest phases and in Fig. 6 as the point of maximum compression is approached. Our explanation for these discrepancies is that: (i) chromatic effects start diluting the emittance as we move approximately $20^{\circ}$ off-crest in the linac; (ii) CSR dominates the emittance growth as the bunch length approaches the point of full compression. Chromatic effects in principle include

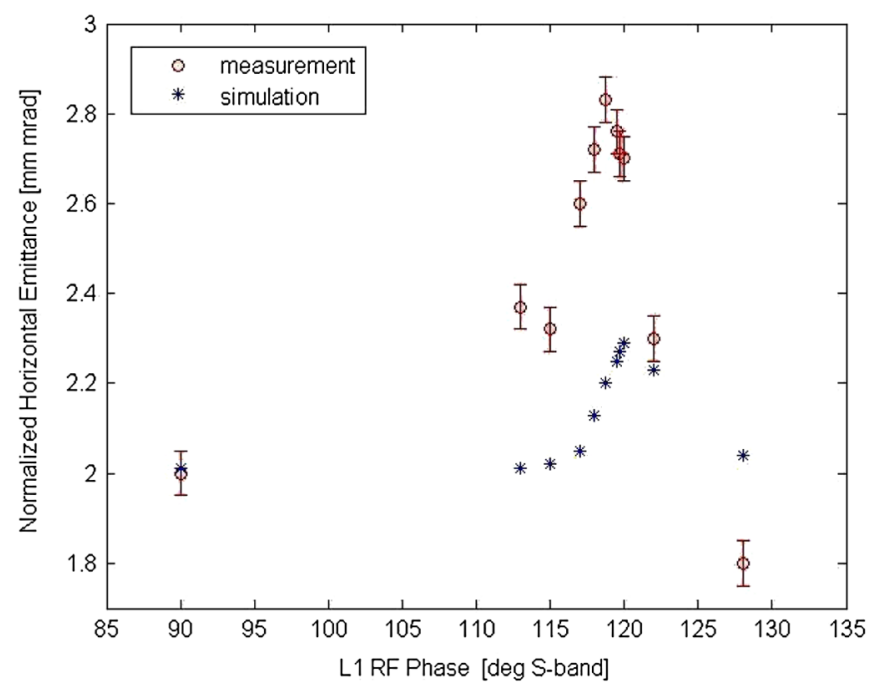

FIG. 6. Comparison of the normalized horizontal emittance measured and simulated as a function of the L1 rf phase. BC1 is set to $85 \mathrm{mrad}$ bending angle. The maximum beam energy occurs at phase $90^{\circ}$ while bunch length compression happens for $\phi>90^{\circ}$. The ELEGANT simulations include both CSR and LSC. 
chromatic aberration and spurious dispersion. The former is excluded a priori because, according to Eq. (3), $\beta_{x}$ would need to be as large as $160 \mathrm{~m}$ in the $\mathrm{BC} 1$ area to explain the discrepancy in Fig. 5. We have no evidences of such a large optics mismatch in this area compared to the design optics. Instead, the discrepancy can be easily explained by means of Eq. (2) if a spurious dispersion of $\sim 4 \mathrm{~mm}$ accumulates by the $\mathrm{BC} 1$ area. This level is a reasonable expectation [34] and might occur, for example, in the presence of bad trajectory steering and/or mishandling of the beam position monitor offsets. To check this possibility, the dispersion is going to be directly and systematically measured along the entire machine in next runs of commissioning. At the same time, the unexpected CSR effect can be explained in terms of beam optics mismatch with respect to the design Twiss parameters. We will discuss this issue in more detail in the next section.

\section{B. Emittance as a function of optics}

We now explore the emittance sensitivity to the optics across $\mathrm{BC} 1$ during compression. The strength of the quadrupole Q_L01.04, located just upstream of BC1, is systematically varied (see Fig. 7) with fixed $C=6.5$ (corresponding to $\phi=115^{\circ}$ in Fig. 6). The design optics foresees $k=1.625 \mathrm{~m}^{-2}$. According to Eq. (5), the CSRinduced relative emittance growth is proportional both to the betatron function in the bending plane and to the square of the CSR-induced energy spread (see also [35]). The

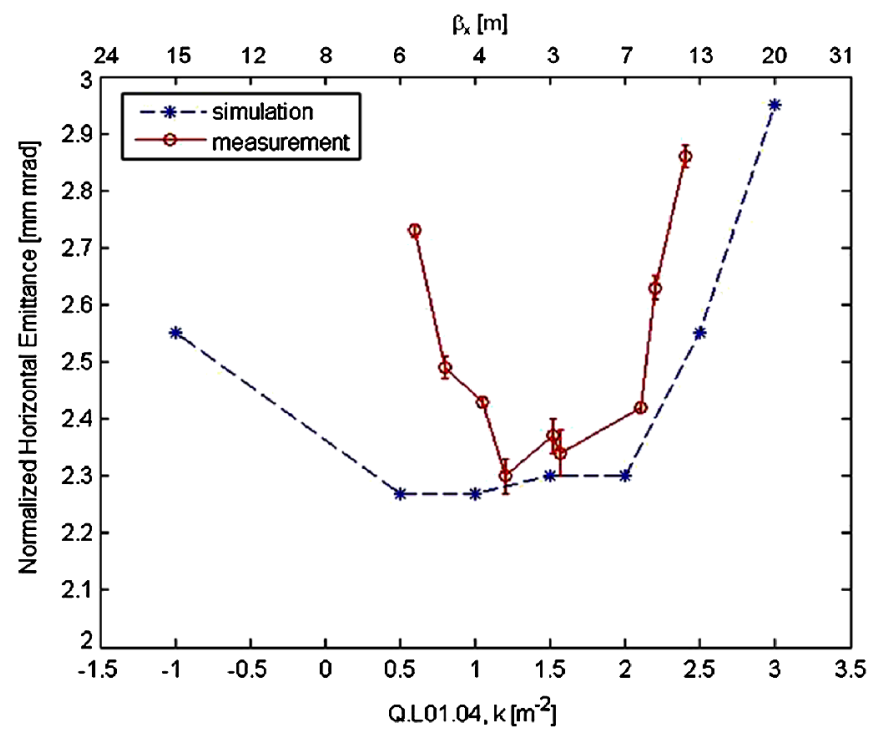

FIG. 7. Comparison of measured and simulated normalized horizontal emittance as a function of the strength $k$ of the quadrupole Q_L01.04 or, equivalently, of the expected $\beta_{x}$ in the middle of the third dipole magnet of $\mathrm{BC} 1$. The design optics foresees $k=1.625 \mathrm{~m}^{-2}$. Other relevant parameters include $C=6.5$ with $\mathrm{BC} 1$ at $85 \mathrm{mrad}$ bending angle and L1 $\mathrm{rf}$ phase at $25^{\circ}$ far from crest. The ELEGANT simulation includes both CSR and LSC effects. latter is in turn inversely proportional to the bunch length [11], which reaches its minimum by the third magnet of the chicane. The global CSR effect is therefore dominated by the energy spread induced in the second half of the chicane and can be minimized by a small $\beta_{x}$ in this region. So, when the quadrupole strength is scanned, we expect a region of $k$ values associated with small $\beta_{x}$ in which $\varepsilon_{N, x}$ reaches a minimum. Our expectations are supported by the experimental and simulation results displayed in Fig. 7. These results are apparently in contrast with those of Refs. [36,37]. However, we notice that both these works report a longitudinal charge density distribution that is at least 10 times greater than ours, due to a bunch charge exceeding $5 \mathrm{nC}$, over a comparable bunch length, with a compression energy $\sim 5$ times smaller. Their bunch parameters lie in the so-called long magnet, short bunch CSR regime, instead of the long bunch regime that applies to Fermi. Thus, we argue that intrabunch space charge forces are probably playing a much more important role in those cases. Space charge forces are indeed expected to blow up the emittance for decreasing beam size due to increased charge density. Such an effect is observed in [37] (e.g., their Fig. 8) where a similar interpretation is given.

Still relying on the validity of Eq. (5) for the Fermi case, we have next verified (see Fig. 8) the underlying correctness of the optics transport from the $\mathrm{LH}$ to the $\mathrm{BC} 1$ area, as a function of the L1 rf phase $\phi$ or, equivalently, of $C$. In fact, as $\phi$ changes, the focusing properties of L1 change as well as the beam energy at each quadrupole location, requiring that the quadrupole strengths be set to the design value in order to keep the optics fixed. This procedure becomes more important as the energy chirp increases to

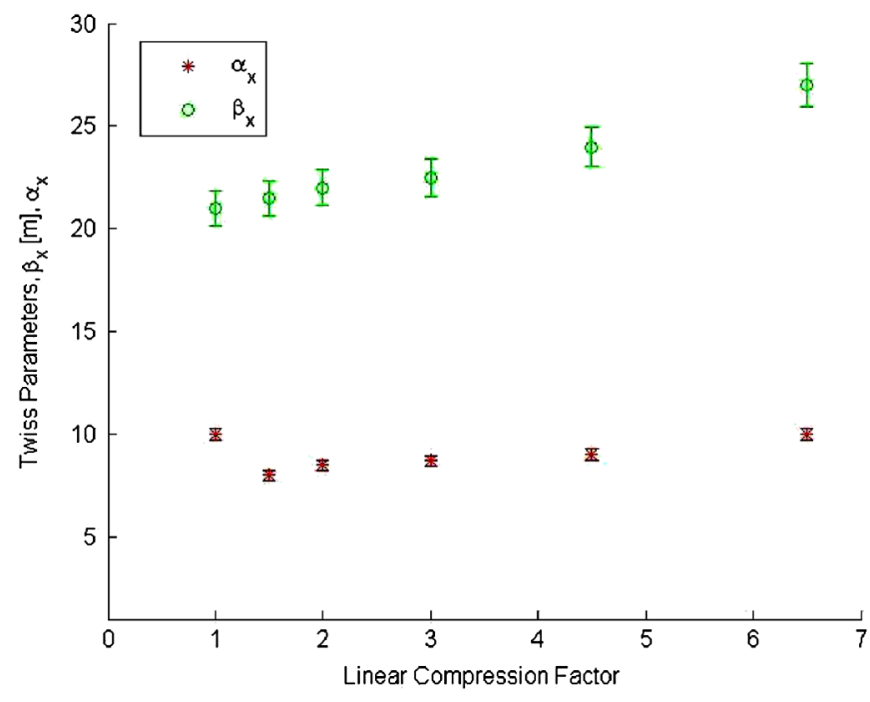

FIG. 8. Beam horizontal Twiss parameters (green circle marker for $\beta_{x}$, red star marker for $\alpha_{x}$ ) measured as a function of the compression factor. This is varied by keeping $\mathrm{BC} 1$ at the fixed bending angle of $85 \mathrm{mrad}$ and by varying the L1 $\mathrm{rf}$ phase. Theoretically, $C=6.5$ corresponds to $\Delta \phi=25^{\circ}$ from the crest. 
achieve a higher $C$ (i.e., $\phi$ approaches the point of zero crossing).

In spite of the agreement shown in Fig. 7 between simulation and measurements around the nominal quadrupole setting, we observe experimentally larger emittance excursions for $k$ varying more than $20 \%$. This suggests a lack of control of the real beam Twiss parameters, confirmed by their variation in Fig. 8. Such an effective mismatch could arise from unexpected focusing contributions and/or slice optics mismatch. Slice optics mismatch could also explain the small emittance oscillation observed for $k$ varying less than $20 \%$ of the nominal value (see the local maximum in Fig. 7).

\section{Emittance preservation}

In order to reduce the emittance growth at moderate compression factors and to improve the beam optics control in $\mathrm{BC} 1$, we have adopted the following approach. Relying on the measurements in Figs. 5 and 6, we assume that for $C \geq 5\left(\Delta \phi \geq 20^{\circ}\right)$ the horizontal emittance behavior is dominated by CSR emission. In this case, we focus our attention on the emittance dependence on the optics inside the chicane. We have then compared the emittance vs compression factor with three different transport optics in the compressor: (a) the nominal design with $\mathrm{BC} 1$ set at the nominal angle of $85 \mathrm{mrad}$, (b) a new design with smaller $\beta_{x}$ inside $\mathrm{BC} 1$ ( $\beta_{x}$ nominally shrinks from 3 to $1 \mathrm{~m}$ in the third dipole of $\mathrm{BC} 1$ and from 6 to $3 \mathrm{~m}$ in the fourth one), and (c) nominal design but with $\mathrm{BC} 1$ set to

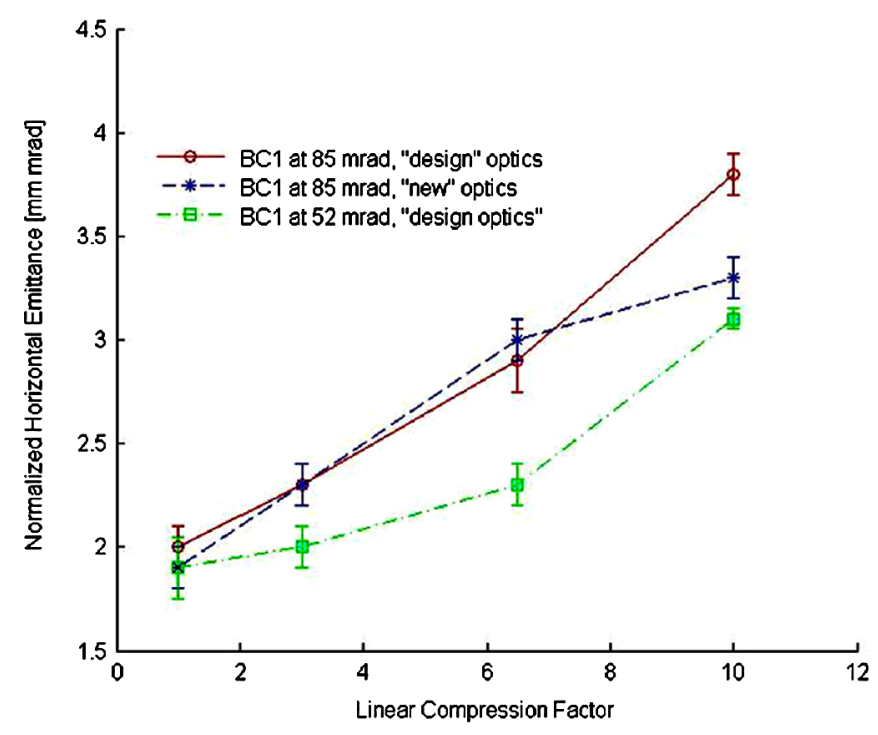

FIG. 9. Normalized horizontal emittance measured as a function of the linear compression factor, $C$. For each line, BC1 is kept fixed while the L1 rf phase is moved far from crest to vary $C$. The lines refer to: original design optics with $\mathrm{BC} 1$ at $85 \mathrm{mrad}$ bending angle (circle marker-solid red line); "new" optics with $\mathrm{BC} 1$ at $85 \mathrm{mrad}$ (circle marker-dashed black line); original design optics with $\mathrm{BC} 1$ at $52 \mathrm{mrad}$ (square marker-dashed green line).

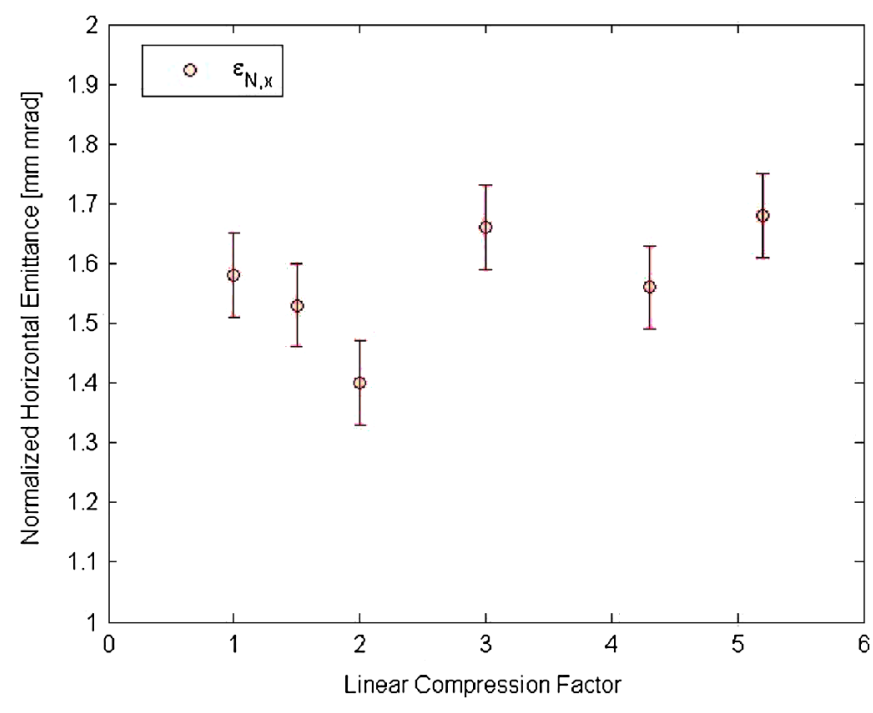

FIG. 10. $\varepsilon_{N}$ measured as a function of the compression factor, which is varied by the $\mathrm{L} 1 \mathrm{rf}$ phase. $\mathrm{BC} 1$ is at $85 \mathrm{mrad}$ bending angle.

$52 \mathrm{mrad}$ (Fig. 9). In the latter case a larger energy chirp (1.5\% instead of $1.0 \%$ ) is provided by L1 to ensure the same compression as in the $85 \mathrm{mrad}$ case. At low compression factors configurations (a) and (b) provide similar results. As expected the advantage of a low horizontal $\beta_{x}$ appears at high compression ratio, $C=10$. The design (c), in spite of the higher energy chirp, reduces the final emittance from $\varepsilon_{N, x}=3.8 \mathrm{~mm} \mathrm{mrad}$ to $\varepsilon_{N, x}=3.1 \mathrm{~mm} \mathrm{mrad}$. These results demonstrate that a careful optics manipulation inside the compressor is essential to minimizing the CSR effect. In addition, the fact that a larger energy chirp (c) does not degrade the final emittance is in agreement with the given interpretation that other mechanisms of emittance growth such as chromatic aberration and spurious dispersion, if present, are small with respect to those due to CSR.

These considerations suggest that the actual beam matching can be improved with Twiss parameters closer to the design values. We carried out careful measurement and matching of the Twiss parameters in the $\mathrm{LH}$ and $\mathrm{BC} 1$ area at each new value of $C$ (or, equivalently, L1 rf phase), in order to include in the matching the variations of the optical properties of the linac and to be as close as possible to the desired nominal values, especially inside $\mathrm{BC} 1$. At the same time, we adopted the optics design (b) with smaller $\beta_{x}$ in the second half of $\mathrm{BC} 1$. Doing this, we have been able to keep $\varepsilon_{N, x}$ below $1.7 \mathrm{~mm}$ mrad up to $C=5.2$, as shown in Fig. 10 with a small dependence of the Twiss parameters on the compression factor, as shown in Fig. 11.

\section{CONCLUSIONS}

The horizontal emittance degradation of a 200 pC, 6 ps duration electron beam has been investigated in the $\mathrm{BC} 1$ area of Fermi@Elettra FEL during one-stage, nonlinear 


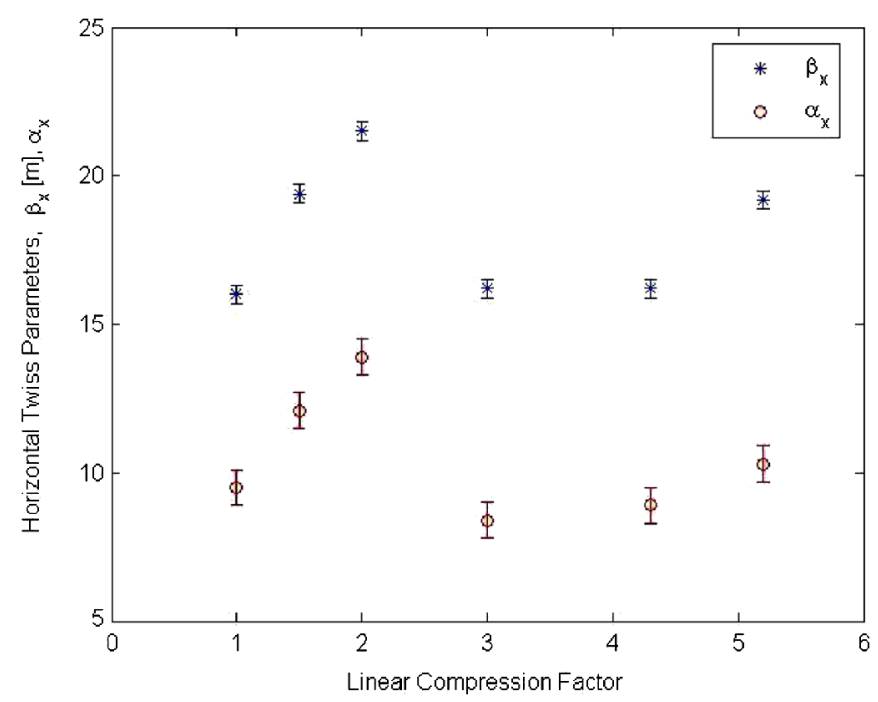

FIG. 11. Beam horizontal Twiss parameters (red circle marker for $\alpha_{x}$, black star marker for $\beta_{x}$ ) measured as a function of the compression factor. This is varied by keeping $\mathrm{BC} 1$ at the fixed bending angle of $85 \mathrm{mrad}$ and by varying the L1 $\mathrm{rf}$ phase.

bunch length compression. Theoretical models, particle tracking, and numerous experiments have been presented, employing many different conditions of magnetic bunch length compression. Emittance values higher than those predicted by theory have been measured for large, off-crest rf phasing at the beginning of commissioning. This discrepancy cannot be completely explained by chromatic aberration, which is excluded by analytical considerations that predict an emittance blowup 1 order of magnitude smaller than that observed experimentally. Instead, we offer an alternative explanation that a spurious dispersion of a few mm generates $\sim 25 \%$ emittance increase when the relative energy spread is $\sigma_{\delta} \approx 1.0 \%$.

Bunch length compression studies have been performed with the one-stage configuration but without linearization of the longitudinal phase space. CSR emission seems to dominate the emittance growth when $C>5$. These results suggested means to minimize horizontal emittance degradation during compression in $\mathrm{BC} 1$. The adoption of optics with smaller $\beta_{x}$ in the second half of $\mathrm{BC} 1$, as well as a smaller bending angle in $\mathrm{BC} 1$ and a larger energy chirp, diminishes the emittance growth for the same compression factor. The best control of the emittance growth has finally been achieved by tuning the beam optics for each individual value of the compression factor. The injector emittance has been preserved for values of $C$ up to 5.2 and the final emittance is smaller than $1.7 \mathrm{~mm}$ mrad. This machine configuration has been adopted to optimize Fermi FEL radiation output at wavelengths in the range 30-60 nm. Building upon these results, our next steps to improve the Fermi electron beam quality will include detailed investigations of the spurious dispersion, slice optics, and transverse wakefield effects.

\section{ACKNOWLEDGMENTS}

The authors thank M. Cornacchia for his suggestions about the implementation of a new optics to reduce the chromatic aberration, G. De Ninno for his support to the emittance measurements, and D. Castronovo for his contribution to the reconstruction of the energy distribution. We also thank M. Venturini and A. A. Zholents for useful discussions concerning the measurement setup and the experimental results reported in this article.

\section{APPENDIX A: RECONSTRUCTION OF THE ENERGY DISTRIBUTION}

The particle energy distribution during compression has been reconstructed experimentally by using a horizontal scraper in the middle of $\mathrm{BC} 1$. This technique has been adopted instead of the more common (and typically easier!) measurement of the beam size in a spectrometer line. In fact, during compression, the $\sim 1$-m dispersion at the screen in the spectrometer line couples to $\sim 1 \%$ energy spread, resulting in a beam size larger than the screen target.

A horizontal scraper, installed in the middle of $\mathrm{BC} 1$, is moved to gradually cut longitudinal portions of the bunch. The scraper is made of two, independently movable cylindrical rods of copper. With a thickness of $13 \mathrm{~mm}$, they absorb most of the charge intercepted with negligible scattering [38]. The dispersive path in $\mathrm{BC} 1$ transforms the particle energy-longitudinal position correlation, $(E, z)$, into a correlation in the spatial coordinates, $(z, x)$. From the operational point of view, L1 is run off-crest by $25^{\circ}$ and $\mathrm{BC} 1$ is set to $85 \mathrm{mrad}$ bending angle, resulting in

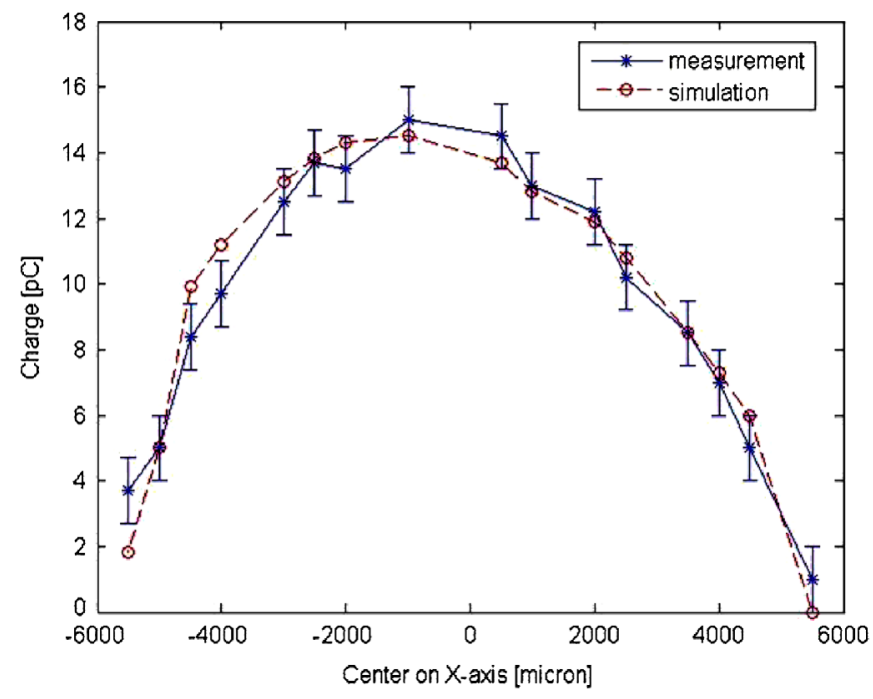

FIG. 12. Charge downstream of $\mathrm{BC} 1$ during compression $(C=6.5)$ vs horizontal scraper position. Two blades of the scraper select an open window of $1 \mathrm{~mm}$. The horizontal particle distribution in the middle of $\mathrm{BC} 1$ reflects the particle energy distribution. 
$C=6.5$. The horizontal dispersion in the middle of $\mathrm{BC} 1$ is $255 \mathrm{~mm}$. First, the particle energy distribution in the middle of $\mathrm{BC} 1$ is mapped by selecting $1 \mathrm{~mm}$ open window between the left and the right blade, where the beam horizontal extension is approximately $12 \mathrm{~mm}$. The total charge transmitted after beam scraping is detected at a charge monitor downstream of $\mathrm{BC} 1$.

Figure 12 shows the charge measured downstream of $\mathrm{BC} 1$ versus the central position of the scraper. Assuming that the charge distribution reflects the particle energy distribution, we find that the FWHM relative energy spread is $4.5 \% \pm 0.1 \%$; the rms energy spread is $0.9 \% \pm 0.1 \%$, in excellent agreement with the simulation result. The charge measurement has an error of about $\pm 5 \mathrm{pC}$. The energy spread uncertainty is dominated by the energy acceptance of the scraper window.

[1] C. Bocchetta et al., FERMI@Elettra FEL Conceptual Design Report No. ST/F-TN-07/12, 2007, http://www .elettra.trieste.it/FERMI/index.php?n=Main.CDRdocument.

[2] L.-H. Yu, M. Babzien, I. Ben-Zvi, L.F. DiMauro, A. Doyuran, W. Graves, E. Johnson, S. Krinsky, R. Malone, I. Pogorelsky, J. Skaritka, G. Rakowsky, L. Solomon, X. J. Wang, M. Woodle, V. Yakimenko, S. G. Biedron, J. N. Galayda, E. Gluskin, J. Jagger, V. Sajaev, and I. Vasserman, Science 289, 932 (2000).

[3] R. Bonifacio, R. Corsini, and P. Pierini, Phys. Rev. A 45, 4091 (1992).

[4] I. Boscolo and V. Stagno, Nuovo Cimento B 58, 267 (1980).

[5] L.-H. Yu, Phys. Rev. A 44, 5178 (1991).

[6] E. M. Allaria, P. Craievich, S. Di Mitri, G. Penco, and M. Trovo, in Proceedings of the SPIE Optics+Optoelectronics Conference 8078: Advances in X-ray Free-Electron Lasers, Prague, CZ (2011).

[7] K. L. F. Bane, F.-J. Decker, Y. Ding, D. Dowell, P. Emma, J. Frisch, Z. Huang, R. Iverson, C. Limborg-Deprey, H. Loos, H.-D. Nuhn, D. Ratner, G. Stupakov, J. Turner, J. Welch, and J. Wu, Phys. Rev. ST Accel. Beams 12, 030704 (2009).

[8] J.S. Nodvick and D.S. Saxon, Phys. Rev. 96, 180 (1954).

[9] R. L. Warnock and P. Morton, Part. Accel. 25, 113 (1990).

[10] J. B. Murphy, S. Krinsky, and R. L. Gluckstern, Part. Accel. 57, 9 (1997).

[11] E. L. Saldin, E. A. Schneidmiller, and M. V. Yurkov, Nucl. Instrum. Methods Phys. Res., Sect. A 398, 373 (1997).

[12] Ya.S. Derbenev, J. Rossbach, E. L. Saldin, and V.D. Shiltsev, Report No. DESY-TESLAFEL-95-05, 1995.

[13] P. Craievich, S. Di Mitri, and A. A. Zholents, Nucl. Instrum. Methods Phys. Res., Sect. A 604, 457 (2009).

[14] D. H. Dowell, Nucl. Instrum. Methods Phys. Res., Sect. A 429, 320 (1999).

[15] H. Braun, F. Chautard, R. Corsini, T. O. Raubenheimer, and P. Tenenbaum, Phys. Rev. Lett. 84, 658 (2000).

[16] H. H. Braun, R. Corsini, L. Groening, F. Zhou, A. Kabel, T. O. Raubenheimer, R. Li, and T. Limberg, Phys. Rev. ST Accel. Beams 3, 124402 (2000).
[17] F. Zhou, A. Kabel, J. Rosenzweig, R. Agustsson, G. Andonian, D. Cline, A. Murokh, and V. Yakimenko, Phys. Rev. ST Accel. Beams 9, 114201 (2006).

[18] Michael Röhrs, Christopher Gerth, Holger Schlarb, Bernhard Schmidt, and Peter Schmüser, Phys. Rev. ST Accel. Beams 12, 050704 (2009).

[19] M. G. Minty and F. Zimmermann, Report No. SLAC-R621, 2003.

[20] R. Akre, D. Dowell, P. Emma, J. Frisch, S. Gilevich, G. Hays, Ph. Hering, R. Iverson, C. Limborg-Deprey, H. Loos, A. Miahnahri, J. Schmerge, J. Turner, J. Welch, W. White, and J. Wu, Phys. Rev. ST Accel. Beams 11, 030703 (2008).

[21] F.-J. Decker, Report No. SLAC-PUB 6684, 1994).

[22] H. Loos, R. Akre, A. Brachmann, F.-J. Decker, Y. Ding, D. Dowell, P. Emma, J. Frisch, S. Gilevich, G. Hays, Ph. Hering, Z. Huang, R. Iverson, C. Limborg-Deprey, A. Miahnahri, S. Molloy, H.-D. Nuhn, J. Turner, J. Welch, W. White, J. Wu, and D. Ratner, in Proceedings of the 30th FEL Conference, Gyeongju, Korea (2008), THBAU01.

[23] M. Borland, Report No. APS LS-287, 2000).

[24] C. Scafuri, in Proceedings of ICALEPCS 2009, Kobe, Japan (2009), TUP037.

[25] http://www.mathworks.com/products/matlab/

[26] M. Sands, Report No. SLAC-AP-85, 1991.

[27] A. W. Chao and M. Tigner, Handbook of Accelerator Physics and Engineering (World Scientific, Singapore, 2006), 3rd ed.

[28] E. L. Saldin, E. A. Schneidmiller, and M. Yurkov, Report No. TESLA-FEL2003-02, 2003.

[29] Z. Huang and K.-J. Kim, Phys. Rev. ST Accel. Beams 5, 074401 (2002).

[30] M. Huning, P. Piot, and H. Schlarb, Nucl. Instrum. Methods Phys. Res., Sect. A 475, 348 (2001).

[31] M. Borland, Phys. Rev. ST Accel. Beams 4, 070701 (2001).

[32] M. Venturini, Phys. Rev. ST Accel. Beams 10, 104401 (2007).

[33] S. Di Mitri, E. Allaria, L. Badano, C. Bontoiu, M. Cornacchia, P. Craievich, M. Danailov, G. DeNinno, B. Diviacco, O. Ferrando, S. Ferry, F. Iazzourene, S. V. Milton, G. Penco, S. Spampinati, M. Trovo, M. Veronese, W. Fawley, S. Lidia, G. Penn, J. Qiang, K. G. Sonnad, M. Venturini, R. Warnock, A. A. Zholents, I. V. Pogorelov, M. Borland, G. Bassi, J. A. Ellison, K. Heinemann, R. Fiorito, A. Shkvarunets, and J. C. Tobin, Nucl. Instrum. Methods Phys. Res., Sect. A 608, 19 (2009).

[34] E. Prat, W. Decking, and T. Limberg, Phys. Rev. ST Accel. Beams 13, 040701 (2010).

[35] F. Stulle, A. Adelmann, and M. Pedrozzi, Phys. Rev. ST Accel. Beams 10, 031001 (2007).

[36] H. H. Braun, S. Dobert, L. Groening, M. Borland, and A. Kabel, in Proceedings of the 19th Particle Accelerator Conference, Chicago, Illinois, 2001 (IEEE, Piscataway, NJ, 2001), p. 164.

[37] R. Talman, N. Malitsky, and F. Stulle, Phys. Rev. ST Accel. Beams 12, 014201 (2009).

[38] S. Ferry, C. Bontoiu, P. Craievich, S. Di Mitri, and E. Karantzoulis, in Proceedings of the 23rd Particle Accelerator Conference, Vancouver, Canada, 2009 (IEEE, Piscataway, NJ, 2009), WE6RFP043. 